\title{
Factores asociados a gonartrosis en pacientes mayores de 40 años atendidos en el Hospital Santa Rosa- 2018
}

Ana Luz Báez Ayala* 1,a; Ingrit Melina Taipe Huamán 1,a; Nora de las Mercedes Espíritu Salazar 2,b

\section{RESUMEN}

Objetivo: Determinar los factores asociados a gonartrosis en pacientes mayores de 40 años del Hospital Santa Rosa en 2018.

Materiales y métodos: Se realizó un estudio analítico de casos y controles (61 casos y 122 controles) de pacientes mayores de 40 años con diagnóstico de gonartrosis y sin diagnóstico de gonartrosis atendidos en el Hospital Santa Rosa en 2018 . En ambos grupos se evaluaron los factores asociados edad, sexo, estado nutricional, hipertensión arterial, diabetes mellitus tipo 2, ocupación, traumatismo de rodilla, lumbalgia, síndrome metabólico y tabaquismo. Para el análisis estadístico se emplearon la prueba de $\mathrm{X}^{2}$ y OR (regresión logística) para el análisis estadístico.

Resultados: El 88,5\% de los pacientes con gonartrosis fue del sexo femenino. De los pacientes con esta enfermedad, el $68,9 \%$ tenían como ocupación ama de casa; el rango de edad más frecuente fue de 60 a 69 en los casos (45,9\%) y 50 a $59(37,7 \%)$ en los controles. Se encontró una asociación significativa entre el riesgo de padecer gonartrosis y el haber presentado lumbalgia (OR = 6,395, IC 95\% 3,163 - 12,929) y el sexo femenino (OR = 3, 905; IC 95\% 1,632 - 9,342). En cuanto al antecedente de traumatismo de rodilla y el tabaquismo, no mostraron significancia.

Conclusiones: Se concluye que el sexo femenino y la lumbalgia están implicados en la aparición y progresión de la gonartrosis. La edad y la ocupación tuvieron una asociación significativa con la presencia de esta enfermedad.

Palabras clave: Osteoartritis; Rodilla; Factores de riesgo; Femenino (Fuente: DeCS BIREME).

\section{Factors associated with gonarthrosis in patients older than 40 years treated at the Hospital Santa Rosa in 2018}

\section{ABSTRACT}

Objective: To determine the factors associated with gonarthrosis in patients older than 40 years of the Hospital Santa Rosa in 2018.

Materials and methods: An analytical, case-control (61 cases and 122 controls) study was carried out in patients older than 40 years of age with and without a diagnosis of gonarthrosis treated at the Hospital Santa Rosa in 2018 . In both groups, associated factors such as age, sex, nutritional status, hypertension, type 2 diabetes mellitus, occupation, knee trauma, low back pain, metabolic syndrome and smoking were evaluated. Pearson's chi-squared test and OR (logistic regression) were used for the statistical analysis.

Results: Eighty-eight point five percent $(88.5 \%)$ of the patients with gonarthrosis were females. Out of the patients with this disease, $68.9 \%$ were housewives; the most frequent age range was 60 to 69 years in the case group (45.9\%) and 50 to 59 years in the control group (37.7\%). A significant association was found between the risk of suffering from gonarthrosis and having suffered low back pain $(\mathrm{OR}=6.395 ; 95 \% \mathrm{Cl}=3.163-12.929)$ and being female $(\mathrm{OR}=3.905 ; 95 \% \mathrm{Cl}=1.632$ 9.342). Regarding the history of knee trauma and smoking, they did not show significance.

Conclusions: It is concluded that females and low back pain are involved in the onset and progression of gonarthrosis. Age and occupation had a significant association with the occurrence of this disease.

Keywords: Osteoarthritis; Knee; Risk factors; Female (Source: MeSH NLM).

1 Universidad de San Martín de Porres, Facultad de Medicina Humana. Lima, Perú.

2 Instituto Nacional de Salud. Lima, Perú.

a Estudiante de Medicina Humana.

b Pediatra salubrista.

*Autor corresponsal. 


\section{INTRODUCCIÓN}

La gonartrosis es una enfermedad inflamatoria crónica, progresiva y degenerativa que afecta especialmente, a la población mayor de 40 años y, en su mayoría, a las mujeres. En las articulaciones se produce una lesión caracterizada por la pérdida progresiva del cartílago articular en la rodilla, aparición de osteofitos y cambios en la membrana sinovial. En la actualidad, se acepta la existencia de un patrón génico y proteómico de características inflamatorias semejantes al de la artritis reumatoide o al síndrome metabólico ${ }^{(1-4)}$. Es considerada la cuarta causa más importante de discapacidad en mujeres, y la octava en los varones ${ }^{(5)}$.

Se considera un problema de salud pública por la severa discapacidad que ocasiona y por los costos que genera en los servicios de salud. La prevalencia es de 25 a $30 \%$ en las personas entre 45 a 65 años; pero en mayores de 65 años, la prevalencia es del $85 \%$. La gonartrosis es la enfermedad más susceptible a riesgos de traumatismos ${ }^{(6)}$.

La literatura señala que la gonartrosis está aumentando en todo el mundo, y se espera que se incremente a $52 \%$ para el 2040 por el envejecimiento y el incremento de peso. En México, se ha previsto que en el año 2050 el total de pacientes con gonartrosis será, en promedio, de 20 millones ${ }^{(6)}$. En España, el $10 \%$ de la población mayor de 60 años tiene una incapacidad funcional severa como consecuencia de la gonartrosis, con una notable repercusión sobre su calidad de vida, lo que se traduce a un incremento de gastos socioeconómicos para el Estado ${ }^{(7)}$.

En el Perú, las cifras de prevalencia de gonartrosis proceden, mayormente, de estadísticas de los establecimientos de salud a los que asisten los pacientes e indican un incremento en la demanda de atención. En la consulta de reumatología, la gonartrosis fue una de las enfermedades que se diagnosticó con mayor frecuencia en el seguro social, y en la práctica privada alcanza el $15 \%$ de las consultas. No existen muchos estudios de la prevalencia de esta enfermedad aunque se han realizado alguno en poblaciones reducidas por lo que sus resultados se consideran como una tendencia aproximada. Sin embargo, estas investigaciones son valiosas porque muestran la realidad peruana. En un estudio piloto, que se realizó en Lima en pacientes mayores de 50 años, Castañeda et al. encontraron una prevalencia de gonartrosis de $18 \%{ }^{(8)}$. En el hospital José Agurto Tello de Chosica (2016), se encontró que el $60 \%$ de los pacientes atendidos en consultorios externos presentó gonartrosis ${ }^{\left({ }^{9}\right.}$.

El impacto social generado por la gonartrosis no solo se refiere al sufrimiento personal, sino también al uso de recursos para la salud. Se prevé que esta enfermedad continúe en aumento debido a algunos factores determinantes como el envejecimiento de la población y la creciente prevalencia de obesidad $(8,10-12)$ lo que ha motivado a plantear este estudio que tiene como objetivo determinar los factores asociados a la gonartrosis en pacientes mayores de 40 años en el Hospital Santa Rosa de Lima, Perú.

\section{MATERIALES Y MÉTODOS}

\section{Diseño y población de estudio}

Corresponde a un estudio cuantitativo, observacional, retrospectivo, transversal y analítico (caso-control). Se comparó a dos grupos: los casos (pacientes mayores de 40 años con diagnóstico de gonartrosis en consulta externa) y los controles (pacientes mayores de 40 años sin diagnóstico de gonartrosis en consulta externa). En ambos grupos se evaluaron los siguientes factores asociados: edad, sexo, estado nutricional, hipertensión arterial (HTA), diabetes mellitus tipo 2 (DM tipo 2), ocupación, traumatismo de rodilla, lumbalgia, síndrome metabólico y tabaquismo. La población de estudio fueron pacientes mayores de 40 años con diagnóstico de gonartrosis y sin diagnóstico de gonartrosis atendidos en el Hospital Santa Rosa en 2018.

\section{Variables y mediciones}

Se usó la fórmula de cálculo del tamaño de la muestra para casos y controles no pareados a través del programa Epi InfoTM, y se calculó un tamaño muestral de 61 casos y 122 controles, lo que permitió alcanzar una potencia del $80 \%$ para detectar un riesgo relativo de 2,67 para una prevalencia del factor estudiado igual a $35 \%$ en el grupo control.

Para el presente estudio se utilizó un muestreo aleatorio randomizado simple con base en a los criterios de inclusión. Se incluyeron como casos a todos los pacientes mayores de 40 años con diagnóstico de gonartrosis en el servicio de Reumatología del Hospital Santa Rosa en el periodo 2018 y con historia clínica completa. Se excluyeron los pacientes con diagnóstico de artrosis en otras articulaciones que no fuera la rodilla, con diagnóstico de artritis reumatoide y con historia clínica incompleta.

Los controles fueron seleccionados del libro de registro de admisión del Servicio de Medicina Interna del mismo hospital, mayores de 40 años y pareados por edad (+/- 5 años). Además, debían ser personas sin diagnostico anterior de gonartrosis.

Se usó una ficha de recolección de datos como instrumento, que tuvo dos segmentos: "Datos generales" (edad, sexo, ocupación, estado civil, etc.), y "Factores asociados" (hipertensión arterial, diabetes mellitus tipo 2, traumatismo de rodilla, etc.). En la ficha de recolección, cada ítem tuvo una codificación para cuestiones posteriores de tabulación y practicidad. 


\section{Análisis estadístico}

Los datos obtenidos de las historias clínicas y fichas de recolección fueron tabulados con el programa Excel y analizados con el programa SPSS versión 24.

Se realizó un análisis univariado, las variables cualitativas se expresaron en frecuencias y porcentajes. Para evaluar la asociación entre cada una de las variables independientes y el desenlace empleamos el cálculo de OR, los intervalos de confianza del $95 \%$ (IC 95\%) y valores de $p$. Se escogieron las variables con mayor asociación y se realizó una regresión logística con el fin de ajustar los OR crudos para disminuir la variable de confusión.

\section{Consideraciones éticas}

El protocolo fue aprobado por el Comité de Ética de la FMH - USMP y el Comité de Investigación del Hospital Santa Rosa. Este trabajo siguió las normas de buena práctica clínica y en todo momento cumplió con las normas éticas. La identidad de cada paciente se codificó para conservar el anonimato. Además, no implicó ningún tipo de riesgo para los pacientes dado que se utilizó solo sus historias clínicas.

\section{RESULTADOS}

Se obtuvo información de 163 pacientes (61 casos y 122 controles). De ellos, 135 fueron mujeres y 48, hombres. El sexo femenino conforma el $88,50 \%(n=54)$ del grupo de casos y el $66,4 \%(n=81)$ del grupo de controles.

El rango de edad que predominó en el grupo de casos fue de 60 a 69 años con un $45,90 \%(n=28)$; y en el grupo de controles fue de 50 a 59 años con un $37,70 \%(n=46)$. Entre los "casos", la ocupación de "ama de casa" tuvo una frecuencia de $68,90 \%(n=42)$, y en los "controles" fue de $28,70 \%(n=35)$. En los casos, el grado de instrucción que predominó fue "secundaria completa" con 44,30\% ( $n=27$ ), y en los controles, fue "superior universitario completa": $34,40 \%(n=42)$. Se encontró que la edad, sexo, estado civil, ocupación y grado de instrucción están estadísticamente asociados con la gonartrosis (Tabla 1).

Tabla 1. Características sociodemográficas de los casos y controles mayores de 40 años (Hospital Santa Rosa, 2018)

\begin{tabular}{|c|c|c|c|c|c|c|}
\hline \multirow{2}{*}{$\begin{array}{c}\text { Características } \\
\text { sociodemográficas }\end{array}$} & \multicolumn{2}{|c|}{ Casos: $n=61$} & \multicolumn{4}{|c|}{ Controles: $n=122$} \\
\hline & $\mathrm{n}$ & $(\%)$ & $\mathrm{n}$ & $(\%)$ & $X^{2}$ & $p$ \\
\hline \multicolumn{7}{|l|}{ Edad } \\
\hline $40-49$ & 5 & $(8,20)$ & 42 & $(34,40)$ & & \\
\hline $50-59$ & 18 & $(29,50)$ & 46 & $(37,70)$ & 24,008 & 0,000 \\
\hline $60-69$ & 28 & $(45,90)$ & 26 & $(21,30)$ & & \\
\hline 70 a más & 10 & $(16,40)$ & 8 & $(6,60)$ & & \\
\hline \multicolumn{7}{|l|}{ Sexo } \\
\hline Masculino & 7 & $(11,50)$ & 41 & $(33,60)$ & & \\
\hline Femenino & 54 & $(88,50)$ & 81 & $(66,40)$ & 10,294 & 0,001 \\
\hline \multicolumn{7}{|l|}{ Estado civil } \\
\hline Soltero & 13 & $(21,30)$ & 49 & $(40,20)$ & & \\
\hline Casado & 31 & $(50,80)$ & 57 & $(46,70)$ & & \\
\hline Viudo & 6 & $(9,80)$ & 1 & $(0,80)$ & 14,2 & 0,006 \\
\hline Divorciado & 4 & $(6,60)$ & 7 & $(5,70)$ & & \\
\hline Conviviente & 7 & $(11,50)$ & 8 & $(6,60)$ & & \\
\hline \multicolumn{7}{|l|}{ Ocupación } \\
\hline Empleado & 10 & $(16,40)$ & 61 & $(50,00)$ & & \\
\hline Independiente & 7 & $(11,50)$ & 21 & $(17,20)$ & & \\
\hline Ama de casa & 42 & $(68,90)$ & 35 & $(28,70)$ & 28,3 & 0,000 \\
\hline Otros & 2 & $(3,30)$ & 5 & $(4,10)$ & & \\
\hline
\end{tabular}




\begin{tabular}{|c|c|c|c|c|c|c|}
\hline \multirow{2}{*}{$\begin{array}{l}\text { Características } \\
\text { sociodemográficas }\end{array}$} & \multicolumn{2}{|c|}{ Casos: $n=61$} & \multicolumn{4}{|c|}{ Controles: $n=122$} \\
\hline & $\mathrm{n}$ & $(\%)$ & $\mathrm{n}$ & $(\%)$ & $x^{2}$ & $p$ \\
\hline \multirow{2}{*}{\multicolumn{7}{|c|}{$\begin{array}{l}\text { Edad } \\
\text { Grado de instrucción }\end{array}$}} \\
\hline & & & & & & \\
\hline Primaria incompleta & 1 & $(1,60)$ & 2 & $(1,60)$ & & \\
\hline Primaria completa & 9 & $(14,80)$ & 3 & $(2,50)$ & & \\
\hline Secundaria incompleta & 3 & $(4,90)$ & 6 & $(4,90)$ & & \\
\hline Secundaria completa & 27 & $(44,30)$ & 41 & $(33,60)$ & & \\
\hline Superior no universitaria incompleta & 0 & $(0,00)$ & 2 & $(1,60)$ & 16,04 & 0,025 \\
\hline Superior no universitaria completa & 6 & $(9,80)$ & 15 & $(12,30)$ & & \\
\hline Superior universitaria incompleta & 2 & $(3,30)$ & 11 & $(9,00)$ & & \\
\hline Superior universitaria completa & 13 & $(21,30)$ & 42 & $(34,40)$ & & \\
\hline
\end{tabular}

En la tabla 2 se presenta el estado nutricional de los casos y controles según las categorías de la Organización Mundial de la Salud (OMS). En los casos se encontró sobrepeso en $41,00 \%(n=25)$ y en los controles $36,10 \%(n=44)$; obesidad en $16,40 \%(n=10)$ de los casos y en $18,9 \%(n=23)$ de los controles; obesidad severa en 1,60\% $(n=1)$ de los casos y en $9,80 \%(n=12)$ de los controles; solamente encontramos seis personas $(4,90 \%)$ con obesidad mórbida en el grupo control. Según el análisis de $X^{2}$ encontramos que la asociación entre estado nutricional y gonartrosis es significativa $\left(X^{2}=8,606\right)$.

Tabla 2. Estado nutricional de los casos y controles mayores de 40 años (Hospital Santa Rosa, 2018)

\begin{tabular}{|c|c|c|c|c|c|c|}
\hline \multirow[t]{2}{*}{ Estado nutricional } & \multicolumn{2}{|r|}{ Casos } & \multicolumn{2}{|c|}{ Controles } & \multirow[b]{2}{*}{$\mathrm{X}^{2}$} & \multirow[b]{2}{*}{$p$} \\
\hline & $\mathrm{n}$ & $(\%)$ & $\mathrm{n}$ & $(\%)$ & & \\
\hline Normal & 25 & $(41,00)$ & 37 & $(30,30)$ & 8,606 & 0,04 \\
\hline Sobrepeso & 25 & $(41,00)$ & 44 & $(36,10)$ & & \\
\hline Obesidad & 10 & $(16,40)$ & 23 & $(18,90)$ & & \\
\hline Obesidad severa & 1 & $(1,60)$ & 12 & $(9,80)$ & & \\
\hline Obesidad mórbida & 0 & $(0,00)$ & 6 & $(4,90)$ & & \\
\hline Total & 61 & $(100,00)$ & 122 & $(100,00)$ & & \\
\hline
\end{tabular}

La tabla 3 muestra el análisis univariado que presenta las variables estudiadas. La lumbalgia se encontró en el 77,00\% de los casos $(n=47)$ y en los controles, en $34,40 \%(n=42)$; síndrome metabólico en $44,30 \%(n=27)$ de los casos y el 23,00\% $(n=28)$ de los controles; mientras que la HTA aparece en el $39,30 \%(n=24)$ de los casos y $20,50 \%$ $(n=25)$ de los controles; la DM tipo 2 se encontró en el $29,50 \%$ de los casos $(n=18)$ y en $13,10 \%(n=16)$ de los controles; el antecedente de traumatismo de rodilla apareció en el 18,00\% $(n=11)$ de los casos y el 9,00\% $(n=11)$ de los controles , y el tabaquismo se presentó en 14,80\% $(n=9)$ de los casos y en el $14,80 \%(n=18)$ de los controles.

Se muestra una asociación significativa entre el riesgo de padecer gonartrosis y el haber presentado lumbalgia (OR = 6, 395; IC 95\%: 3,163-12,929), síndrome metabólico $(O R=2,666 ;$ IC 95\%:1,38 - 5,149), hipertensión arterial (OR = 2,517; IC 95\%: 1,280 - 4,949), DM tipo 2 (OR = 2,773; IC 95\%: 1,296 - 5,936). El antecedente de traumatismo de rodilla y el tabaquismo no mostraron significancia. 
Factores asociados a gonartrosis en pacientes mayores de 40 años atendidos en el Hospital Santa Rosa- 2018

Tabla 3. Factores asociados a gonartrosis de los casos y controles mayores de 40 años (Hospital Santa Rosa, 2018)

\begin{tabular}{lccrrrr} 
& \multicolumn{2}{l}{ Casos: $\mathrm{n}=61$} & Controles: $\mathrm{n}=122$ & OR & IC (95\%) \\
Factores & $\mathrm{n}$ & $(\%)$ & $\mathrm{n}$ & $(\%)$ & & \\
\hline Lumbalgia & 47 & $(77,00)$ & 42 & $(34,40)$ & 6,395 & $3,163-12,929$ \\
\hline Síndrome metabólico & 27 & $(44,30)$ & 28 & $(23,00)$ & 2,666 & $1,38-5,149$ \\
\hline Hipertensión arterial & 24 & $(39,30)$ & 25 & $(20,50)$ & 2,517 & $1,280-4,949$ \\
\hline Diabetes mellitus & 18 & $(29,50)$ & 16 & $(13,10)$ & 2,773 & $1,296-5,936$ \\
\hline Traumatismo de rodilla & 11 & $(18,00)$ & 11 & $(9,00)$ & 2,220 & $0,903-5,460$ \\
\hline Tabaquismo & 9 & $(14,80)$ & 18 & $(14,80)$ & 1,00 & $0,420-2,379$
\end{tabular}

Así mismo, se encontró que el sexo femenino era un factor de riesgo de gonartrosis (OR = 3, 905; IC 95\%: 1,632 - 9,342) con una asociación estadísticamente significativa (Tabla 4).

Tabla 4. Sexo de los casos y controles mayores de 40 años (Hospital Santa Rosa, 2018)

\begin{tabular}{lrrrrrr} 
Sexo & \multicolumn{2}{c}{ Casos } & \multicolumn{2}{c}{ Controles } & OR & IC (95\%) \\
& $\mathrm{n}$ & \multicolumn{1}{c}{$(\%)$} & \multicolumn{1}{c}{$\mathrm{n}$} & \multicolumn{1}{c}{$(\%)$} & & \\
\hline Femenino & 54 & 88,50 & 81 & 66,40 & & \\
\hline Masculino & 7 & 41,00 & 41 & 33,60 & 3,905 & $1,632-9,342$ \\
\hline Total & 61 & 100,00 & 122 & 100,00 & & \\
\hline
\end{tabular}

Según el análisis de regresión logística, la lumbalgia y el sexo resultaron estadísticamente significativos $(p<0,05)($ Tabla 5).

Tabla 5. Regresión logística de los factores asociados a gonartrosis de los casos y controles en mayores de 40 años (Hospital Santa Rosa, 2018)

\begin{tabular}{lcccrrr} 
& & & \multicolumn{2}{c}{$\operatorname{Exp(B)}$} & \multicolumn{2}{c}{ IC 95\% para EXP(B) } \\
Factores & B & Sig. & \multicolumn{1}{c}{ OR } & Inferior & Superior \\
\hline Sexo & 1,510 & 0,002 & 4,525 & 1,744 & 11,740 \\
\hline Hipertensión arterial & 0,659 & 0,118 & 1,933 & 0,846 & 4,417 \\
\hline Diabetes mellitus & 0,528 & 0,287 & 1,696 & 6,41 & 4,484 \\
\hline Síndrome metabólico & 0,243 & 0,569 & 1,275 & 5,53 & 2,939 \\
\hline Dolor lumbar & 1,844 & 0,000 & 6,321 & 2,971 & 13,449 \\
Constante & $-3,304$ & 0,000 & 0,037 & & \\
\hline
\end{tabular}




\section{DISCUSIÓN}

La gonartrosis es una enfermedad frecuente en nuestro hospital. Este estudio contó con 183 participantes, los cuales presentaron los factores de riesgo asociados. Encontramos como factores de riesgo el sexo femenino $(\mathrm{OR}=3,905$; IC 95\%: $1,632-9,342$ ) y la lumbalgia (OR = 6,395; IC 95\%: $3,163-12,929)$. El síndrome metabólico $(O R=2,666$; IC 95\%: 1,38-5,149), hipertensión arterial ( $O R=2,517$ IC $95 \%: 1,280-4,949)$ y diabetes mellitus tipo $2(O R=2,773$; IC 95\%: 1,296 - 5,936) no mostraron significancia en el análisis de la regresión logística.

Se ha descrito que la frecuencia de gonartrosis predomina en el sexo femenino, como lo demostraron Lee et al. al referir una relación de 2,07: 1 con el sexo masculino ${ }^{(13)}$, lo que apoya nuestros resultados debido a que identificamos una relación de 7,71: 1 respectivamente; asimismo, detectamos una diferencia estadísticamente significativa entre los casos y controles $(p<0,01)$.

Coincidimos con Mena R. en que la gonartrosis está relacionado con la edad; prevaleció en el grupo con edad de 60 años a más (49\%), y en el de 50 a 59 años $(33,3 \%)^{(14)}$. Mientras que en nuestro estudio se encontró que el rango de edad más frecuente fue de 60 a 69 años en el $45,90 \%$ en los casos, y en el grupo de 50 a 59 años $(37,70 \%)$ en los controles.

El artículo publicado por Rojas S. establece que uno de los factores de riesgo modificables es la ocupación, ya que se observó que la gonartrosis predomina en los trabajos que requieren el uso prolongado y repetitivo de ciertas articulaciones (rodilla), y esto se relaciona con ser ama de casa en $45 \%{ }^{(15)}$. En esta investigación encontramos que el $68,90 \%$ los pacientes con gonartrosis eran amas de casa. Respecto al estado civil, nuestro estudio muestra que el $50,80 \%$ de los pacientes está casado. En el trabajo de Zhang et al. se encontró que los residentes de la provincia de Shanxi divorciados o viudos tuvieron una mayor prevalencia de gonartrosis que los solteros o los casados ${ }^{(16)}$. Esta diferencia puede deberse a que el tamaño muestral es mayor $(n=7126)$, lo mismo que el rango de edad (16 años a más) en comparación a nuestro estudio.

Lee et al. plantearon que el bajo nivel educativo (primaria incompleta, primaria completa, secundaria incompleta) estaba asociado con el incremento del riesgo de gonartrosis ${ }^{(17)}$. Por el contrario, en nuestro trabajo se halló que la educación secundaria completa y superior universitaria son los grados de educación más frecuentes en los pacientes con gonartrosis. Esto podría ser debido al sistema educativo que se otorga y a que los datos obtenidos fueron de una encuesta nacional.

En relación al índice de masa corporal, en ambas poblaciones (casos y controles) se observó que el estado nutricional que predominó fue el sobrepeso ( $41,00 \%$ en los casos y $36,10 \%$ en los controles) lo cual difiere con lo señalado por CharlesLozoya et al. que identificaron la obesidad como estado nutricional más frecuente $(63,9 \%$ en los casos y $55,7 \%$ en los controles) ${ }^{(18)}$. Estas diferencias pueden deberse a que el primer estudio se realizó en un Servicio de Unidad Médica de Alta Especialidad, y en nuestro hospital de estudio acuden pacientes con diferentes comorbilidades.

El rol de la diabetes mellitus en el desarrollo de la gonartrosis es cada vez más notable, Charles-Lozoya et al. obtuvieron que la diabetes mellitus tipo 2 (OR = 2,1; IC 95\%: 1,1 - 4), la hipertensión arterial $(\mathrm{OR}=1,9 ; \mathrm{IC} 95 \%: 1,1-3,3)$ y el síndrome metabólico (OR = 2,6; IC 95\%: 1,4 - 4,7) se comportaron como factores de riesgo ${ }^{(18)}$. En nuestro estudio, en el análisis multivariado se halló que la diabetes mellitus tipo 2 (OR de 2,773 IC 95\% 1,296-5,936), hipertensión arterial ( $O R=2,517$; IC 95\%: 1,280 - 4,949) y síndrome metabólico $(O R=2,666$; IC $95 \%: 1,38-5,149$ ) eran factores asociados a riesgo de gonartrosis; sin embargo, al realizar la regresión logística no se demostró dicha asociación.

Suri $\mathrm{P}$ et al. mostraron que el $57,4 \%$ de los pacientes con gonartrosis presentaron lumbalgia (asociación estadísticamente significativa) (19). De igual manera, al realizar el análisis de regresión logística se pudo comprobar que el $77 \%$ de los casos de gonartrosis estuvieron asociados con lumbalgia $O R=6,395$; IC $95 \%: 3,163-12,929$ ).

Moghimi et al. encontraron que uno de los factores de riesgo para gonartrosis fue haber sufrido un traumatismo de rodilla $(O R=2,28 I C=1,03-2,96)^{(20)}$. Nuestro trabajo no demostró que este antecedente sea un factor de riesgo $(O R=2,22 ; \mathrm{IC}=0,90-5,46)$, debido a que nuestro tamaño muestral fue menor y los datos del dicho estudio se obtuvieron de un programa de control de enfermedades reumáticas.

Según Arellano et al., el tabaquismo se asoció con la gonartrosis como factor de protección $(\mathrm{OR}=0,78$; IC :0,5 - 1,2); mientras que en nuestro estudio se encontró que el tabaquismo se comportó como factor de riesgo, aunque esta asociación no fue estadísticamente significativa $(O R=1,0 ; I C=0,4-2,3)$ dado que la población tiene diferentes hábitos y costumbres ${ }^{(21)}$.

El estudio fue diseñado para investigar factores de riesgo de gonartrosis, los pacientes se seleccionaron de los servicios de Reumatología y Medicina Interna. Una de las limitaciones de nuestro estudio fue la diferencia entre las edades de los casos y los controles, el rango de edad más frecuente en los casos fue de 60 a 69 años, y en los controles fue de 50 a 59 años. A pesar de esto, se puede inferir cuales son los factores de riesgo que están asociados a la gonartrosis. Además, los criterios estrictos de selección y el uso de un muestreo aleatorio simple pueden compensar 
las limitaciones de este artículo.

Se sugiere realizar un estudio con un mayor tamaño de muestra, teniendo en cuenta los factores que evidenciaron una tendencia a ser de riesgo pero que no mostraron significancia al realizar el análisis de regresión logística como el síndrome metabólico, la hipertensión arterial y la diabetes mellitus tipo 2 . Además, se recomienda usar un instrumento de recolección (encuesta) y que el trabajo sea prospectivo para poder evaluar otros factores asociados a la gonartrosis.

Se concluye en este estudio que el sexo femenino y la lumbalgia están implicados en la aparición y progresión de la gonartrosis. La edad y la ocupación tuvieron una asociación significativa con la presencia de esta enfermedad.

Es importante identificar los factores de riesgo en una persona con gonartrosis, sobre todo los modificables, con el propósito de promover actividades de prevención de esta enfermedad degenerativa que afecta la calidad de vida.

Contribuciones de los autores: Ana Luz Báez Ayala e Ingrit Melina Taipe Huamán realizaron conjuntamente y en partes iguales la concepción y diseño del artículo, recolección y obtención de los datos, así como la redacción del artículo. La magister Nora Espíritu Salazar, nuestra asesora, realizó la revisión crítica y la aprobación de la versión final del artículo.

Fuentes de financiamiento: El artículo ha sido financiado por los autores.

Conflicto de interés: Los autores declaran no tener ningún conflicto de interés.

\section{REFERENCIAS BIBLIOGRÁFICAS}

1. Martínez Figueroa R, Martínez Figueroa C, Calvo Rodríguez R, Figueroa Poblete D. Osteoartritis (artrosis) de rodilla. Rev Chil Ortop Traumatol. 2015; 56(3): 45-51.

2. O'Neill TW, McCabe PS, McBeth J. Update on the epidemiology, risk factors and disease outcomes of osteoarthritis. Best Pract Res Clin Rheumatol. 2018; 32(2): 312-26.

3. Niu J, Clancy M, Aliabadi P, Vasan R, Felson DT. Metabolic syndrome, its components, and knee osteoarthritis: the Framingham osteoarthritis study. Arthritis Rheumatol. 2017; 69(6): 1194-203.

4. Favero M, Ramonda R, Goldring MB, Goldring SR, Punzi L. Early knee osteoarthritis. RMD Open. 2015; 1(Suppl. 1): e000062.

5. Bravo Acosta T, Téllez Díaz Z, Hernández Tápanes S, Pedroso Morales I, Martín Cordero JE, Fernández Cuesta JI. Calidad de vida relacionada con la salud en adultos mayores con gonartrosis. Invest Medicoquir. 2015; 7(1): 59-76.

6. Villarreal-Ríos E, Cedillo-García M, Vargas-Daza ER, GaliciaRodríguez L, Martínez-González L, Escorcia-Reyes V. Costo directo de la atención médica en pacientes con gonartrosis. Reumatol
Clín. 2019; 15(5): 277-81.

7. Cortés Izquierdo MJ. Gonartrosis, presentación de un caso y revisión de la literatura [Tesis de pregrado]. Soria: Universidad de Valladolid. Escuela Universitaria de Fisioterapia; 2013.

8. Castañeda O, Kuroiwa R, Torres D, Castañeda A, Manche-Kuroiwa S, Priori E. Evaluación de la eficacia y seguridad del ácido hialurónico por vía oral asociado con glucosamina sulfato, condroitín sulfato y metilsulfonilmetano comparado con la asociación por vía oral de glucosamina sulfato, condroitín sulfato y metilsulfonilmetano en la osteoartritis de rodilla. Acta Méd Peruana. 2014; 31(3): 157-64.

9. Vargas Campana CA. Prevalencia y factores de riesgo asociados a osteoartritis en pacientes atendidos por consultorio externo del hospital José Agurto Tello de Chosica [Tesis de pregrado]. Lima: Universidad Ricardo Palma. Facultad de Medicina Humana; 2018.

10. National Center for Biotechnology Information. Obesity - MeSH - NCBI [Internet]. Disponible en: https://www.ncbi.nlm.nih.gov/ mesh/?term=obesity

11. Deshpande BR, Katz JN, Solomon DH, Yelin EH, Hunter DJ, Messier $\mathrm{SP}$, et al. Number of persons with symptomatic knee osteoarthritis in the us: impact of race and ethnicity, age, sex, and obesity. Arthritis Care Res. 2016; 68(12): 1743-50.

12. Misra D, Fielding RA, Felson DT, Niu J, Brown C, Nevitt M, et al. Risk of knee osteoarthritis with obesity, sarcopenic obesity, and sarcopenia. Arthritis Rheumatol. 2019; 71(2): 232-7.

13. Lee S, Kim S-J. Prevalence of knee osteoarthritis, risk factors, and quality of life: the Fifth Korean National Health and Nutrition Examination Survey. Int J Rheum Dis. 2017; 20(7): 809-17.

14. Mena Pérez R. Caracterización de pacientes con gonartrosis de rodilla. Centro de diagnóstico integral "concepción”. Rev Haban Cienc Méd. 2016; 15(1): 17-26.

15. Rojas Cordova SA. Factores de riesgo asociados a la presencia de artrosis en sujetos mayores de 50 años de la comunidad de Chuma. Cientifica. 2014; 12(1): 41-5.

16. Zhang J-F, Song L-H, Wei J-N, Zhang A-L, Dong H-Y, Wen H-Y, et al. Prevalence of and risk factors for the ocurrence of symptomatic in rural regions of Shanxi Province, China. Int J Rheum Dis. 2016; 19(8): 781-9.

17. Lee S, Kwon Y, Lee N, Bae K-J, Kim J, Park S, et al. The prevalence of osteoarthritis and risk factors in the Korean Population: the Sixth Korea National Health and Nutrition Examination Survey (VI1, 2013). Korean J Fam Med. 2019; 40(3): 171-5.

18. Charles-Lozoya S, Treviño-Báez JD, Ramos-Rivera JA, RangelFlores JM, Tamez-Montes JC, Brizuela-Ventura JM. Síndrome metabólico y otros factores asociados a gonartrosis. Gac Med Méx. 2017; 153(7): 775-80.

19. Suri P, Morgenroth DC, Kwoh CK, Bean JF, Kalichman L, Hunter DJ. Low back pain and other musculoskeletal pain comorbidities in individuals with symptomatic osteoarthritis of the knee: data from the osteoarthritis initiative. Arthritis Care Res (Hoboken). 2010; 62(12): 1715-23.

20. Moghimi N, Rahmani K, Delpisheh A, Saidi A, Azadi NA, Afkhamzadeh A. Risk factors of knee osteoarthritis: a case-control study. Pak J Med Sci. 2019; 35(3): 636-40.

21. Arellano Pérez Vertti RD, Arguello Astorga JR, Hernández Terán F, García Salcedo JJ. Factores de riesgo en osteartrosis de rodilla en una población mexicana de casos y controles. Rev Cubana Ortop Traumatol. 2013; 27(1): 22-32. 
Correspondencia:

Ana Luz Báez Ayala

Dirección: Jr. San Antonio Mz. M Lte. 15, Urb. Residencial-

Surco.

Teléfono: 982324259

Correo electrónico: ani47_96@hotmail.com

Recibido: 22 de noviembre de 2019

Evaluado: 27 de julio de 2020

Aprobado: 18 de agosto de 2020

( ) La revista. Publicado por Universidad de San Martín de Porres, Perú. (cc) Br Licencia de Creative Commons Artículo en acceso abierto bajo términos de Licencia Creative Commons Atribución 4.0 Internacional. (http://creativecommons.org/licenses/by/4.0/)

ORCID iDs

Ana Luz Báez Ayala C https://orcid.org/0000-0001-7472-0471

Ingrit Melina Taipe Huamán C https://orcid.org/0000-0002-1151-538X

Nora de las Mercedes Espíritu S. ㄱ https://orcid.org/0000-0002-6340-02851 